\title{
Pacific
}

Journal of

Mathematics

\section{BERGMAN AND HARDY SPACES WITH SMALL EXPONENTS}

KEHE ZHU 


\title{
BERGMAN AND HARDY SPACES WITH SMALL EXPONENTS
}

\section{KeHE ZHU}

\begin{abstract}
We show that for each $0<p<1$ the dual space of the Hardy and weighted Bergman space on the open unit ball is isomorphic to the Bloch space (with equivalent norms) under certain volume integral pairing.
\end{abstract}

1. Introduction. We present a new approach to an old problem, namely, the problem of describing the continuous linear functionals on the Bergman and Hardy spaces with $0<p<1$. We restrict our attention to the open unit ball in $\mathbf{C}^{n}$, even though our approach has the potential to generalize to bounded symmetric domains.

Let $B_{n}$ be the open unit ball in $\mathbf{C}^{n}$ with boundary $\partial B_{n}$. Let $H\left(B_{n}\right)$ denote the space of all holomorphic functions in $B_{n}$. For $0<p<+\infty$ and $\alpha>-1$ we let

$$
L_{a}^{p}\left(B_{n}, d v_{\alpha}\right)=H\left(B_{n}\right) \cap L^{p}\left(B_{n}, d v_{\alpha}\right)
$$

denoted the weighted Bergman space, where

$$
d v_{\alpha}(z)=C_{\alpha}\left(1-|z|^{2}\right)^{\alpha} d v(z) .
$$

Here $d v$ is volume measure on $B_{n}$ and $C_{\alpha}$ a normalizing constant so that $d v_{\alpha}$ has total mass 1 . For $f \in L_{a}^{p}\left(B_{n}, d v_{\alpha}\right)$ we write

$$
\|f\|_{\alpha, p}=\left[\int_{B_{n}}|f(z)|^{p} d v_{\alpha}(z)\right]^{1 / p} .
$$

A linear functional $F$ on $L_{a}^{p}\left(B_{n}, d v_{\alpha}\right)$ is bounded if there exists a constant $C>0$ such that $|F(f)| \leq C\|f\|_{\alpha, p}$ for all $f$ in $L_{a}^{p}\left(B_{n}, d v_{\alpha}\right)$. The dual space of $L_{a}^{p}\left(B_{n}, d v_{\alpha}\right)$, denoted $L_{a}^{p}\left(B_{n}, d v_{\alpha}\right)^{*}$, consists of all bounded linear functionals on $L_{a}^{p}\left(B_{n}, d v_{\alpha}\right)$. For each $0<p<+\infty$ the space $L_{a}^{p}\left(B_{n}, d v_{\alpha}\right)^{*}$ is a Banach space with the norm

$$
\|F\|=\sup \left\{|F(f)|:\|f\|_{\alpha, p} \leq 1\right\} .
$$

Note that $L_{a}^{p}\left(B_{n}, d v_{\alpha}\right)$ itself is not a Banach space when $0<p<1$. 
For $0<p<+\infty$ we let $H^{p}\left(B_{n}\right)$ denote the Hardy space consisting of holomorphic functions $f$ in $B_{n}$ such that

$$
\|f\|_{H^{p}}=\sup _{0<r<1}\left[\int_{\partial B_{n}}|f(r w)|^{p} d \sigma(w)\right]^{1 / p}<+\infty
$$

where $d \sigma$ is the normalized Euclidean measure on $\partial B_{n}$. For $0<$ $p<+\infty$ we let $H^{p}\left(B_{n}\right)^{*}$ denote the dual space of $H^{p}\left(B_{n}\right)$ consisting of linear functionals $F$ on $H^{p}\left(B_{n}\right)$ such that

$$
\|F\|=\sup \left\{|F(f)|:\|f\|_{H^{p}} \leq 1\right\}<+\infty .
$$

Again $H^{p}\left(B_{n}\right)^{*}$ is a Banach space with the above norm for each $0<$ $p<+\infty$, even though $H^{p}\left(B_{n}\right)$ itself is not a Banach space when $0<p<1$.

The dual space of $H^{p}\left(B_{n}\right)$ for $0<p<1$ has been studied by several authors. In the one dimensional case Romberg [8] described the dual of $H^{p}$ for all $p \in(0,1)$ except $p=1 /(k+1)$ (where $k$ is any positive integer). Duren, Romberg, and Shields [5] completed the characterization of the dual of $H^{p}$ for all $0<p<1$ in the case $n=1$. They described the dual of $H^{p}$ of the unit disk in terms of the Lipschitz spaces and the Zygmund class. These results were then generalized to the polydisk by Frazier [6] and to the unit ball by Hahn and Mitchell [7]. The basic ideas and constructions in these papers are somewhat similar. See [4] for more information on $H^{p}$ of the disk with $0<p<1$.

Shapiro [10] gave a different and quite general approach to the duality problem of Bergman and Hardy spaces with $0<p<1$. The results in [10] were stated and proved for the disk, but it is clear that some techniques and ideas there work in more general situations. Shapiro's approach, based on the notion of Mackey topologies, is more geometric.

Coifman and Rochberg [2] also considered the duality problem for weighted Bergman spaces with small exponents. Their arguments were based on the theory of atomic decomposition for certain classes of holomorphic functions.

Our formulation of the duality and the method of approach are different from the ones mentioned above, the main difference being in the duality pairing. Let $\mathscr{B}\left(B_{n}\right)$ denote the Bloch space of $B_{n}$ consisting of holomorphic functions $f$ in $B_{n}$ with

$$
\|f\|_{\mathscr{B}}=|f(0)|+\sup \left\{\left(1-|z|^{2}\right)|\nabla f(z)|: z \in B_{n}\right\}<+\infty,
$$


where $\nabla f(z)=\left(\partial f(z) / \partial z_{1}, \ldots, \partial f(z) / \partial z_{n}\right)$ is the complex gradient of $f$ at $z$, and

$$
|\nabla f(z)|=\left[\sum_{k=1}^{n}\left|\frac{\partial f}{\partial z_{k}}(z)\right|^{2}\right]^{1 / 2} .
$$

It is well known that $\mathscr{B}\left(B_{n}\right)$ is a Banach space with the above norm. We describe the dual spaces of $L_{a}^{p}\left(B_{n}, d v_{\alpha}\right)$ and $H^{p}\left(B_{n}\right)$ for $0<p<$ 1 in terms of the Bloch functions. Our main results are Theorems 5 and 6 , stated below as Theorems $\mathrm{A}$ and $\mathrm{B}$, respectively.

Theorem A. Suppose $0<p \leq 1, \alpha>-1$, and $\beta=(n+1+\alpha) / p-$ $(n+1)$. Then the dual space of $L_{a}^{p}\left(B_{n}, d v_{\alpha}\right)$ is isomorphic to $\mathscr{B}\left(B_{n}\right)$ (with equivalent norms) under the duality pairing

$$
\begin{aligned}
\langle f, g\rangle=\lim _{r \rightarrow 1^{-}} \int_{B_{n}} f(r z) \overline{g(z)}\left(1-|z|^{2}\right)^{\beta} d v(z), & \\
& f \in L_{a}^{p}\left(B_{n}, d v_{\alpha}\right), \quad g \in \mathscr{B}\left(B_{n}\right) .
\end{aligned}
$$

Theorem B. Suppose $0<p<1$ and $\beta=(n / p)-(n+1)$. Then the dual of $H^{p}\left(B_{n}\right)$ is isomorphic to $\mathscr{B}\left(B_{n}\right)$ (with equivalent norms) under the duality pairing

$$
\begin{array}{r}
\langle f, g\rangle=\lim _{r \rightarrow 1^{-}} \int_{B_{N}} f(r z) \overline{g(z)}\left(1-|z|^{2}\right)^{\beta} d v(z), \\
f \in H^{p}\left(B_{n}\right), \quad g \in \mathscr{B}\left(B_{n}\right) .
\end{array}
$$

It is likely that experts in this field could deduce the above results from those obtained in [5], [6], [7], [10]. Nevertheless, we think a direct proof here is more natural and desirable.

The author thanks P. Duren, B. Korenblum, S. Krantz, D. Luecking, R. Rochberg, and J. Shapiro for their interest, criticism, and pointing out additional references after the first version of the paper was circulated.

2. A class of fractional derivatives and integrals. In this section we introduce a certain type of radial fractional derivatives and integrals. Recall that $H\left(B_{n}\right)$ is the space of all holomorphic functions in $B_{n}$. We equip $H\left(B_{n}\right)$ with the topology of "uniform convergence on compact sets". Thus a linear operator $T$ on $H\left(B_{n}\right)$ is continuous if and only if $T f_{k} \rightarrow T f$ uniformly on compact sets whenever $f_{k} \rightarrow f$ uniformly on compact sets. For $f \in H\left(B_{n}\right)$ and $0<r<1$ we define $f_{r}$ in $H\left(B_{n}\right)$ by $f_{r}(z)=f(r z)$. 
THEOREM 1. For each $\alpha>-1$ there exists a unique linear operator $D^{\alpha}$ on $H\left(B_{n}\right)$ with the following properties:

(1) $D^{\alpha}$ is continuous on $H\left(B_{n}\right)$.

(2) $D^{\alpha} f_{r}(z)=D^{\alpha} f(r z)$ for all $f \in H\left(B_{n}\right), 0<r<1$, and $z \in B_{n}$.

(3) $D_{z}^{\alpha}\left[(1-\langle z, w\rangle)^{-(n+1)}\right]=C_{\alpha}(1-\langle z, w\rangle)^{-(n+1+\alpha)}$ for each $w \in$ $B_{n}$.

Proof. We first prove uniqueness. Given $f$ in $H\left(B_{n}\right)$ and $0<r<1$ we can write

$$
f_{r}(z)=\int_{B_{n}} \frac{f_{r}(w) d v(w)}{(1-\langle z, w\rangle)^{n+1}}, \quad z \in B_{n}
$$

The above integral converges uniformly for $z$ in any compact subset of $B_{n}$. Thus properties (1) and (3) imply that

$$
\begin{aligned}
D^{\alpha} f_{r}(z) & =\int_{B_{n}} D_{z}^{\alpha}\left[(1-\langle z, w\rangle)^{-(n+1)}\right] f_{r}(w) d v(w) \\
& =C_{\alpha} \int_{B_{n}} \frac{f_{r}(w) d v(w)}{(1-\langle z, w\rangle)^{n+1+\alpha}} .
\end{aligned}
$$

This proves the uniqueness of $D^{\alpha}$.

To prove existence we first define $D^{\alpha}: H^{\infty}\left(B_{n}\right) \rightarrow H\left(B_{n}\right)$ by the formula

$$
D^{\alpha} f(z)=C_{\alpha} \int_{B_{n}} \frac{f(w) d v(w)}{(1-\langle z, w\rangle)^{n+1+\alpha}}, \quad z \in B_{n} .
$$

This along with the reproducing property of the Bergman kernel shows that property (3) holds. Also using homogeneous expansions we easily obtain $D^{\alpha} f_{r}(z)=D^{\alpha} f(r z)$ for each $f \in H^{\infty}\left(B_{n}\right)$. Now for a general $f$ in $H\left(B_{n}\right)$ we define $D^{\alpha} f$ as follows. For each $z \in B_{n}$ there exists $r \in(0,1)$ and $\tilde{z} \in B_{n}$ such that $z=r \tilde{z}$. We define $D^{\alpha} f(z)=$ $D^{\alpha} f_{r}(\tilde{z})$. (Note that $f_{r} \in H^{\infty}\left(B_{n}\right)$ and hence $D^{\alpha} f_{r}$ is defined.) To see that $D^{\alpha} f$ is well-defined, suppose $z=r_{1} \tilde{z}_{1}=r_{2} \tilde{z}_{2}$ with $r_{1}<r_{2}$, say $r_{1}=r r_{2}$ for some $r \in(0,1)$. Since $f_{r_{2}}$ is in $H^{\infty}\left(B_{n}\right)$ we have

$$
D^{\alpha} f_{r_{1}}\left(\tilde{z}_{1}\right)=D^{\alpha} f_{r r_{2}}\left(\tilde{z}_{1}\right)=D^{\alpha} f_{r_{2}}\left(r \tilde{z}_{1}\right)=D^{\alpha} f_{r_{2}}\left(\tilde{z}_{2}\right) \text {. }
$$

Thus $D^{\alpha} f$ is well-defined. Furthermore, $D^{\alpha} f$ is holomorphic since for each $r \in(0,1)$ we have

$$
D^{\alpha} f(r z)=D^{\alpha} f_{r}(z)=C_{\alpha} \int_{B_{n}} \frac{f_{r}(w) d v(w)}{(1-\langle z, w\rangle)^{n+1+\alpha}}, \quad z \in B_{n}
$$


Thus $D^{\alpha}$ as defined above maps $H\left(B_{n}\right)$ to $H\left(B_{n}\right)$. The linearity of $D^{\alpha}$ is obvious. Property (2) follows easily from the definition of $D^{\alpha}$. It remains to show that $D^{\alpha}$ is continuous on $H\left(B_{n}\right)$.

Let $f_{k} \rightarrow f$ in $H\left(B_{n}\right)$. It is clear that for each $r \in(0,1)$ we have $f_{k}(r z) \rightarrow f(r z)$ uniformly for $z \in B_{n}$ as $k \rightarrow+\infty$. Thus for each $r \in(0,1)$ and $k \rightarrow+\infty$ we have

$$
\begin{aligned}
D^{\alpha} f_{k}(r z) & =C_{\alpha} \int_{B_{n}} \frac{f_{k}(\sqrt{r} w) d v(w)}{(1-\langle\sqrt{r} z, w\rangle)^{n+1+\alpha}} \\
& \rightarrow C_{\alpha} \int_{B_{n}} \frac{f(\sqrt{r} w) d v(w)}{(1-\langle\sqrt{r} z, w\rangle)^{n+1+\alpha}} \\
& =D^{\alpha} f(r z)
\end{aligned}
$$

uniformly for $z \in B_{n}$. Since $r$ is arbitrary, we see that $D^{\alpha} f_{k} \rightarrow D^{\alpha} f$ uniformly on each compact subset of $B_{n}$. This completes the proof of Theorem 1 .

REMARK. The proof of the above theorem shows that for each $\alpha>$ -1 the operator $D^{\alpha}$ is given by

$$
D^{\alpha} f(z)=C_{\alpha} \lim _{r \rightarrow 1^{-}} \int_{B_{n}} \frac{f(r w) d v(w)}{(1-\langle z, w\rangle)^{n+1+\alpha}}, \quad f \in H\left(B_{n}\right), \quad z \in B_{n} .
$$

If $f$ in $H\left(B_{n}\right)$ is integrable with respect to volume measure, then it is clear that

$$
D^{\alpha} f(z)=C_{\alpha} \int_{B_{n}} \frac{f(w) d v(w)}{(1-\langle z, w\rangle)^{n+1+\alpha}}, \quad z \in B_{n} .
$$

THEOREM 2. For each $\alpha>-1$ the operator $D^{\alpha}$ is invertible on $H\left(B_{n}\right)$.

Proof. For each $\alpha>-1$ define an operator $D_{\alpha}$ on $H\left(B_{n}\right)$ as follows.

$D_{\alpha} f(z)=\lim _{r \rightarrow 1^{-}} \int_{B_{n}} \frac{\left(1-|w|^{2}\right)^{\alpha} f(r w) d v(w)}{(1-\langle z, w\rangle)^{n+1}}, \quad f \in H\left(B_{n}\right), \quad z \in B_{n}$.

Using arguments similar to those in the proof of Theorem 1 we can show that each $D_{\alpha}$ is a well-defined continuous linear operator on $H\left(B_{n}\right)$. Moreover, by Fubini's theorem and Proposition 7.1.2 of [9] we easily check that $D^{\alpha} D_{\alpha} f=D_{\alpha} D^{\alpha} f$ for all $f \in H^{\infty}\left(B_{n}\right)$. By the continuity of $D^{\alpha}$ and $D_{\alpha}$ and the density of $H^{\infty}\left(B_{n}\right)$ in $H\left(B_{n}\right)$ we conclude that $D_{\alpha}$ is the inverse of $D^{\alpha}$ on $H\left(B_{n}\right)$ for each $\alpha>-1$. 
REMARK. If $\alpha>-1$ and $f$ is in $L_{a}^{1}\left(B_{n}, d v_{\alpha}\right)$, then we have

$$
D_{\alpha} f(z)=\int_{B_{n}} \frac{\left(1-|w|^{2}\right)^{\alpha} f(w) d v(w)}{(1-\langle z, w\rangle)^{n+1}}, \quad z \in B_{n} .
$$

We call $D^{\alpha}$ a radial fractional differential operator of order $\alpha$ and $D_{\alpha}$ a radial fractional integral operator of order $\alpha$. Note that when $-1<\alpha<0$ "integration" here is really "differentiation" and vice versa.

3. Weighted Bergman spaces. In this section we characterize the dual of $L_{a}^{p}\left(B_{n}, d v_{\alpha}\right)$ for each $\alpha>-1$ and $0<p \leq 1$. Recall that

$$
\|f\|_{\alpha, p}=\left[\int_{B_{n}}|f(z)|^{p} d v_{\alpha}(z)\right]^{1 / p}, \quad f \in L_{a}^{p}\left(B_{n}, d v_{\alpha}\right)
$$

Also recall that for $\alpha>-1$ the operator $P_{\alpha}$ is given by

$$
P_{\alpha} f(z)=\int_{B_{n}} \frac{f(w) d v_{\alpha}(w)}{(1-\langle z, w\rangle)^{n+1+\alpha}}, \quad z \in B_{n} .
$$

The proof of the next lemma is due to D. Luecking.

LEMMA 3. For each $0<p \leq 1$ and $\alpha>-1$ there exists a constant $C>0$ such that

$$
\int_{B_{n}}|f(z)|\left(1-|z|^{2}\right)^{(n+1+\alpha) / p-(n-1)} d v(z) \leq C\|f\|_{\alpha, p}
$$

for all $f$ in $L_{a}^{p}\left(B_{n}, d v_{\alpha}\right)$.

Proof. Fix $z$ in $B_{n}$ and let $D(z)$ be the open Euclidean ball in $\mathbf{C}^{n}$ with center $z$ and radius $(1-|z|) / 2$. By the subharmonicity of $|f|^{p}$ we have

$$
|f(z)|^{p} \leq \frac{1}{v(D(z))} \int_{D(z)}|f(w)|^{p} d v(w) .
$$

Since $1-|w| \sim 1-|z|$ for $w \in D(z)$ and $v(D(z)) \sim\left(1-|z|^{2}\right)^{n+1}$, it is easy to find a constant $C_{1}>0$ such that

$$
|f(z)| \leq C_{1}\left(1-|z|^{2}\right)^{-(n+1+\alpha) / p}\|f\|_{\alpha, p}
$$

for all $f$ in $L_{a}^{p}\left(B_{n}, d v_{\alpha}\right)$ and $z \in B_{n}$. For $0<p \leq 1$ we can write $|f(z)|=|f(z)|^{p}|f(z)|^{1-p}$ and estimate the second factor using the above inequality. This easily produces the desired result. 
LEMMA 4. Suppose $\alpha>-1$ and $f$ is holomorphic in $B_{n}$. If either $f$ or the function $\left(1-|z|^{2}\right)^{-\alpha} f(z)$ is bounded on $B_{n}$, then the function $\left(1-|z|^{2}\right)^{\alpha} D^{\alpha} f(z)$ is in $L^{1}\left(B_{n}, d v\right)$ and

$$
\int_{B_{n}} f(z) \overline{g(z)} d v(z)=\int_{B_{n}} D^{\alpha} f(z) \overline{g(z)}\left(1-|z|^{2}\right)^{\alpha} d v(z)
$$

for all $g$ in $H^{\infty}\left(B_{n}\right)$.

Proof. That the function $\left(1-|z|^{2}\right)^{\alpha} D^{\alpha} f(z)$ is in $L^{1}\left(B_{n}, d v\right)$ follows from the definition of $D^{\alpha}$ and 1.4.10 of [9]. The desired result then follows from Fubini's theorem and the fact that the operator $P_{\alpha}$ reproduces bounded holomorphic functions (see 7.1.2 of [9]). (Note that the applicability of Fubini's theorem here also follows from 1.4.10 of [9] and the assumption about $f$.)

Theorem 5. Suppose $0<p \leq 1, \alpha>-1$, and $\beta=(n+1+\alpha) / p-$ $(n+1)$. Then we have $L_{a}^{p}\left(B_{n}, d v_{\alpha}\right)^{*} \simeq \mathscr{B}\left(\boldsymbol{B}_{n}\right)$ with

$$
\begin{aligned}
\langle f, g\rangle=\lim _{r \rightarrow 1^{-}} \int_{B_{n}} f(r z) \overline{g(z)}\left(1-|z|^{2}\right)^{\beta} d v(z), & \\
f & \in L_{a}^{p}\left(B_{n}, d v_{\alpha}\right), \quad g \in \mathscr{B}\left(B_{n}\right) .
\end{aligned}
$$

Proof. Fix $0<p \leq 1, \alpha>-1$, and $F \in L_{a}^{p}\left(B_{n}, d v_{\alpha}\right)^{*}$. Since $\left\|f-f_{r}\right\|_{\alpha, p} \rightarrow 0$ as $r \rightarrow 1^{-}$, we have $F(f)=\lim _{r \rightarrow 1^{-}} F\left(f_{r}\right)$ for all $f \in L_{a}^{p}\left(B_{n}, d v_{\alpha}\right)$. Write

$$
f_{r}(z)=\int_{B_{n}} \frac{f_{r}(w) d v(w)}{(1-\langle z, w\rangle)^{n+1}}, \quad z \in B_{n} .
$$

Since the above integral converges in $L_{a}^{p}\left(B_{n}, d v_{\alpha}\right)$, the continuity of $F$ implies that

$$
F\left(f_{r}\right)=\int_{B_{n}} f_{r}(w) F_{z}\left[\frac{1}{(1-\langle z, w\rangle)^{n+1}}\right] d v(w) .
$$

Let

$$
h(w)=\overline{F_{z}\left[\frac{1}{(1-\langle z, w\rangle)^{n+1}}\right]}, \quad w \in B_{n} .
$$

Then $h$ is holomorphic in $B_{n}$ and

$$
F\left(f_{r}\right)=\int_{B_{n}} f_{r}(w) \overline{h(w)} d v(w) .
$$


It follows from the boundedness of $F$ on $L_{a}^{p}\left(B_{n}, d v_{\alpha}\right)$ and 1.4 .10 of [9] that Lemma 4 can be applied to obtain (The case $\beta=0$ does not require the use of Lemma 4.)

$$
F\left(f_{r}\right)=\int_{B_{n}} f_{r}(w) \overline{D^{\beta} h(w)}\left(1-|w|^{2}\right)^{\beta} d v(w)
$$

where

$$
\beta=\frac{n+1+\alpha}{p}-(n+1) \text {. }
$$

By Property (3) of Theorem 1 we have

$$
D^{\beta} h(w)=C_{\beta} \overline{F_{z}\left[\frac{1}{(1-\langle z, w\rangle)^{(n+1+\alpha) / p}}\right]} .
$$

Let $g=D^{\beta} h$. Then

$$
F\left(f_{r}\right)=\int_{B_{n}} f_{r}(w) \overline{g(w)}\left(1-|w|^{2}\right)^{\beta} d v(w)
$$

and

$$
\frac{\partial g}{\partial w_{k}}(w)=\frac{(n+1+\alpha) C_{\beta}}{p} \overline{F_{z}\left[\frac{z_{k}}{(1-\langle z, w\rangle)^{(n+1+\alpha) / p+1}}\right]}, \quad w \in B_{n} .
$$

Using 1.4.10 of [9] and the boundedness of $F$ on $L_{a}^{p}\left(B_{n}, d v_{\alpha}\right)$ we easily see that $g$ is in the Bloch space $\mathscr{B}\left(B_{n}\right)$.

On the other hand, if $g$ is in $\mathscr{B}\left(B_{n}\right)$, we shall show that the formula

$$
F(f)=\lim _{r \rightarrow 1^{-}} \int_{B_{n}} f_{r}(z) \overline{g(z)}\left(1-|z|^{2}\right)^{\beta} d v(z), \quad f \in L_{a}^{p}\left(B_{n}, d v_{\alpha}\right)
$$

well defines a bounded linear functional on $L_{a}^{p}\left(B_{n}, d v_{\alpha}\right)$, where $\beta=$ $(n+1+\alpha) / p-(n+1)$. By [1] there exists a function $\varphi \in L^{\infty}\left(B_{n}\right)$ such that

$$
g(z)=P_{\beta} \varphi(z)=\int_{B_{n}} \frac{\varphi(w) d v_{\beta}(w)}{(1-\langle z, w\rangle)^{n+1+\beta}}, \quad z \in B_{n} .
$$

Using Fubini's theorem and the reproducing property of $P_{\beta}$ we easily obtain

$$
\int_{B_{n}} f_{r}(z) \overline{g(z)}\left(1-|z|^{2}\right)^{\beta} d v(z)=\int_{B_{n}} f_{r}(w) \overline{\varphi(w)}\left(1-|w|^{2}\right)^{\beta} d v(w)
$$

By Lemma 3 we have

$$
\begin{aligned}
F(f) & =\lim _{r \rightarrow 1^{-}} \int_{B_{n}} f_{r}(z) \overline{g(z)}\left(1-|z|^{2}\right)^{\beta} d v(z) \\
& =\int_{B_{n}} f(z) \overline{\varphi(z)}\left(1-|z|^{2}\right)^{\beta} d v(z)
\end{aligned}
$$


with $|F(f)| \leq C\|\varphi\|_{\infty}\|f\|_{\alpha, p}$ for all $f$ in $L_{a}^{p}\left(B_{n}, d v_{\alpha}\right)$.

4. Hardy spaces $H^{p}\left(B_{n}\right)$ with $0<p<1$. In this section we describe the bounded linear functionals on the Hardy space $H^{p}\left(B_{n}\right)$ with $0<p<1$. The result and method are similar to those in the previous section.

TheOREM 6. Suppose $0<p<1$ and $\alpha=n / p-(n+1)$. Then $H^{p}\left(B_{n}\right)^{*} \simeq \mathscr{B}\left(B_{n}\right)$ with

$$
\begin{aligned}
\langle f, g\rangle=\lim _{r \rightarrow 1^{-}} \int_{B_{n}} f(r z) \overline{g(z)} & \left(1-|z|^{2}\right)^{\alpha} d v(z), \\
& f \in H^{p}\left(B_{n}\right), \quad g \in \mathscr{B}\left(B_{n}\right) .
\end{aligned}
$$

Proof. For $0<p<1$ it is clear that $\alpha=n / p-(n+1)>-1$. If $g$ is in $\mathscr{B}\left(B_{n}\right)$ then by [1] there exists $\varphi \in L^{\infty}\left(B_{n}\right)$ such that $g=P_{\alpha} \varphi$. Writing $g$ as an integral, applying Fubini's theorem, and using the reproducing property of $P_{\alpha}$, we see that

$$
\int_{B_{n}} f_{r}(z) \overline{g(z)}\left(1-|z|^{2}\right)^{\alpha} d v(z)=\int_{B_{n}} f_{r}(z) \overline{\varphi(z)}\left(1-|z|^{2}\right)^{\alpha} d v(z)
$$

for each $f \in H^{p}\left(B_{n}\right)$ and $r \in(0,1)$. By Theorem 11 of [7] there exists a constant $C>0$ such that

$$
\int_{B_{n}}|f(z)|\left(1-|z|^{2}\right)^{\alpha} d v(z) \leq C\|f\|_{H^{p}}
$$

for all $f$ in $H^{p}\left(B_{n}\right)$. This clearly shows that

$$
\begin{aligned}
F(f) & =\lim _{r \rightarrow 1^{-}} \int_{B_{n}} f(r z) \overline{g(z)}\left(1-|z|^{2}\right)^{\alpha} d v(z) \\
& =\int_{B_{n}} f(z) \overline{\varphi(z)}\left(1-|z|^{2}\right)^{\alpha} d v(z), \quad f \in H^{p}\left(B_{n}\right),
\end{aligned}
$$

defines a bounded linear functional on $H^{p}\left(B_{n}\right)$.

Conversely, if $F$ is a bounded linear functional on $H^{p}\left(B_{n}\right)$, then by the first few lines of the proof of Theorem 5 we have $F(f)=$ $\lim _{r \rightarrow 1^{-}} F\left(f_{r}\right)$ and

$$
F\left(f_{r}\right)=\int_{B_{n}} f_{r}(w) \overline{h(w)} d v(w)
$$

where

$$
h(w)=\overline{F_{z}\left[\frac{1}{(1-\langle z, w\rangle)^{n+1}}\right]}, \quad w \in B_{n}
$$


If $\alpha \neq 0$, then 1.4 .10 of [9] and the boundedness of $F$ on $H^{p}\left(B_{n}\right)$ imply that either $h$ is bounded in $B_{n}$ or there exists a constant $C>0$ such that $|h(w)| \leq C\|F\|\left(1-|w|^{2}\right)^{\alpha}$ for all $w \in B_{n}$. Thus we can apply Lemma 4 to obtain

$$
F\left(f_{r}\right)=\int_{B_{n}} f_{r}(w) \overline{g(w)}\left(1-|w|^{2}\right)^{\alpha} d v(w)
$$

where

$$
g(w)=D^{\alpha} h(w)=C_{\alpha} \overline{F_{z}\left[\frac{1}{(1-\langle z, w\rangle)^{n / p}}\right]}, \quad w \in B_{n} .
$$

This is obviously true if $\alpha=0$. For each $1 \leq k \leq n$ we have

$$
\frac{\partial g}{\partial w_{k}}(w)=\frac{n C_{\alpha}}{p} \overline{F_{z}\left[\frac{z_{k}}{(1-\langle z, w\rangle)^{n / p+1}}\right]}, \quad w \in B_{n} .
$$

It follows easily from 1.4.10 of [9] and the boundedness of $F$ on $H^{p}\left(B_{n}\right)$ that there exists a constant $C>0$ such that

$$
\left(1-|w|^{2}\right)\left|\frac{\partial g}{\partial w_{k}}(w)\right| \leq C\|F\|
$$

for all $w$ in $B_{n}$ and $1 \leq k \leq n$. Thus $g$ is in $\mathscr{B}\left(B_{n}\right)$ and the proof of Theorem 6 is completed.

5. Further remarks. In order to generalize our results to bounded symmetric domains we need to answer the following questions:

(1) Does each weighted Bergman projection $P_{\alpha}$ map $L^{\infty}(\Omega)$ onto the same space?

(2) If the answer to the above question is affirmative, then how to describe the space $P_{\alpha} L^{\infty}(\Omega)$ in terms of partial derivatives?

In the case of the polydisk we can settle the above questions satisfactorily. It is proved in [11] that each $P_{\alpha}$ maps $L^{\infty}$ of the polydisk onto the same space $X$, which consists of holomorphic functions $f$ on the polydisk such that

$$
\left(\prod_{k=1}^{n}\left(1-\left|z_{k}\right|^{2}\right)\right) \frac{\partial^{m} f}{\partial z_{i_{1}} \cdots \partial z_{i_{m}}}(z)
$$

is bounded on the polydisk for each $0 \leq m \leq n$ and $1 \leq i_{1}<\cdots<$ $i_{m} \leq n$. Using this result it is then shown in [11] that the dual space of each weighted Bergman space and Hardy space with $0<p<1$ on the polydisk is isomorphic to the above space $X$ (with equivalent norms) under certain integral pairing over the polydisk. Note that the 
duality problem for both the weighted Bergman space and the Hardy space of the polydisk with $0<p<1$ were also studied in [3] and [6].

\section{REFERENCES}

[1] B. R. Choe, Projections, the weighted Bergman spaces, and the Bloch space, Proc. Amer. Math. Soc., 108 (1990), 127-136.

[2] R. Coifman and R. Rochberg, Representation theorems for holomorphic and harmonic functions in $L^{p}$, Astérisque, 77 (1980), 11-66.

[3] A. E. Djrbashian and F. A. Shamoian, Topics in the Theory of $A_{\alpha}^{p}$ Spaces,

B. G. Teubner Verlagsgesellschaft, Leipzig, 1988.

[4] P. L. Duren, Theory of $H^{p}$ Spaces, Academic Press, New York, 1970.

[5] P. L. Duren, B. W. Romberg and A. L. Shields, Linear functionals on $H^{p}$ spaces with $0<p<1$, J. Reine Angew. Math., 238 (1969), 32-60.

[6] A. P. Frazier, The dual space of $H^{p}$ of the polydisk for $0<p<1$, Duke Math. J., 39 (1972), 369-379.

[7] K. T. Hahn and J. Mitchell, Representation of linear functionals in $H^{p}$ spaces over bounded symmetric domains, J. Math. Anal. Appl., 56 (1976), 379-396.

[8] B. W. Romberg, The $H^{p}$ spaces with $0<p<1$, Doctoral dissertation, University of Rochester, 1960.

[9] W. Rudin, Function Theory in the Unit Ball of $\mathbf{C}^{n}$, Springer, New York, 1980.

[10] J. Shapiro, Mackey topologies, reproducing kernels, and diagonal maps on the Hardy and Bergman spaces, Duke Math. J., 43 (1976), 187-202.

[11] K. Zhu, Weighted Bergman projections on the polydisk, to appear in Houston J. Math.

Received November 20, 1991 and in revised form October 20, 1992. Research supported by the National Science Foundation.

STATE UNIVERSITY OF NeW YORK

AlBANY, NY 12222

E-mail address: kz258@albnyvms.bitnet 



\title{
PACIFIC JOURNAL OF MATHEMATICS
}

Founded by

\author{
E. F. BeCKenBACH (1906-1982) F. Wolf (1904-1989)
}

\section{EDITORS}

Sun-Yung A. Chang

(Managing Editor)

University of California

Los Angeles, CA 90024-1555

chang@math.ucla.edu

\section{F. Michael Christ}

University of California

Los Angeles, CA 90024-1555

christ@math.ucla.edu

Herbert Clemens

University of Utah

Salt Lake City, UT 84112

clemens@math.utah.edu
THOMAS ENRIGHT

University of California, San Diego

La Jolla, CA 92093

tenright@ucsd.edu

Nicholas ERCOLANI

University of Arizona

Tucson, AZ 85721

ercolani@math.arizona.edu

R. FINN

Stanford University

Stanford, CA 94305

finn@gauss.stanford.edu

VAUghan F. R. Jones

University of California

Berkeley, CA 94720

vfr@math.berkeley.edu
STEVEN KERCKHOFF

Stanford University

Stanford, CA 94305

spk@gauss.stanford.edu

Martin ScharLemanN University of California Santa Barbara, CA 93106 mgscharl@math.ucsb.edu

Harold Stark

University of California, San Diego La Jolla, CA 92093

V. S. VARADARAJAN University of California

Los Angeles, CA 90024-1555

vsv@math.ucla.edu

\section{SUPPORTING INSTITUTIONS}

UNIVERSITY OF ARIZONA

UNIVERSITY OF BRITISH COLUMBIA

CALIFORNIA INSTITUTE OF TECHNOLOGY

UNIVERSITY OF CALIFORNIA

UNIVERSITY OF MONTANA

UNIVERSITY OF NEVADA, RENO

NEW MEXICO STATE UNIVERSITY

OREGON STATE UNIVERSITY
UNIVERSITY OF OREGON

UNIVERSITY OF SOUTHERN CALIFORNIA

STANFORD UNIVERSITY

UNIVERSITY OF HAWAII

UNIVERSITY OF UTAH

WASHINGTON STATE UNIVERSITY

UNIVERSITY OF WASHINGTON 


\title{
PACIFIC JOURNAL OF MATHEMATICS
}

\author{
Volume $162 \quad$ No. $1 \quad$ January 1994
}

Semisimplicity of restricted enveloping algebras of Lie superalgebras $\quad 1$

JEFFERY MARC BERGEN

A classification of certain 3-dimensional conformally flat Euclidean hypersurfaces

OSCAR J. GARAY

Braided groups of Hopf algebras obtained by twisting

D. GUREVICH and SHAHN MAJID

Flat connections, geometric invariants and the symplectic nature of the 45 fundamental group of surfaces

K. GURUPRASAD

Spin models for link polynomials, strongly regular graphs and Jaeger's 57 Higman-Sims model

PIERRE DE LA HARPE

On the uniqueness of representational indices of derivations of $C^{*}$-algebras

EDWARD KISSIN

On infinitesimal behavior of the Kobayashi distance

MYUNG YULL PANG

Vertex operator construction of standard modules for $A_{n}^{(1)}$

MIRKO PRIMC

Bergman and Hardy spaces with small exponents

KEHE ZHU 